\title{
SOME PROPERTIES OF COMPLEX QUATERNION AND COMPLEX SPLIT QUATERNION MATRICES
}

\author{
Y. ALAGÖZ AND G. ÖZYURT
}

Received 05 March, 2018

\begin{abstract}
The aim of this study is to investigate some properties of complex quaternion and complex split quaternion matrices. To verify this, we use $2 \times 2$ complex matrix representation of these quaternions. Moreover, we present a method to find the determinant of complex quaternion and complex split quaternion matrices. Finally, we research some special matrices for quaternions above.
\end{abstract}

2010 Mathematics Subject Classification: 15B33; 11R52

Keywords: complex quaternion matrix, complex split quaternion matrix

\section{INTRODUCTION}

The real quaternion algebra $\mathbf{H}$ is a four dimensional vector space over the real number field $\mathbb{R}$ and $e_{0}, e_{1}, e_{2}, e_{3}$ denote the basis of $\mathbf{H}$ and basis of $\mathbb{R}^{4}$. The set of real quaternions are a number system that extends the complex numbers field $\mathbb{C}$. Irish mathematician Sir William Rowan Hamilton introduced it in 1843, which is represented as

$$
\mathbf{H}=\left\{a=a_{0} e_{0}+a_{1} e_{1}+a_{2} e_{2}+a_{3} e_{3}: a_{0}, a_{1}, a_{2}, a_{3} \in \mathbb{R}\right\}
$$

where $e_{0}$ acts an identity and $e_{1}^{2}=e_{2}^{2}=e_{3}^{2}=e_{1} e_{2} e_{3}=-1$. Since $e_{2} e_{3} \neq e_{3} e_{2}$ it is obvious that the real quaternions are noncommutative and differ from complex numbers and real numbers. Furthermore any real quaternion can be respesented by a $2 \times 2$ complex matrix, [2]. A complex quaternion it is called also biquaternion $q$ can be written as $q=a_{0} e_{0}+a_{1} e_{1}+a_{2} e_{2}+a_{3} e_{3}$ where $a_{0}, a_{1}, a_{2}, a_{3} \in \mathbb{C}$ and its basis elements $e_{0}, e_{1}, e_{2}, e_{3}$ satisfy the real quaternion multiplication rules. In [5] and [7] conjugates, $2 \times 2$ complex matrices corresponding to basis elements of complex quaternions are expressed.

In 1849, James Cockle introduced the set of real split quaternions which is represented as

$$
\mathbf{H}_{\mathbf{S}}=\left\{p=b_{0} e_{0}+b_{1} e_{1}+b_{2} e_{2}+b_{3} e_{3}: b_{0}, b_{1}, b_{2}, b_{3} \in \mathbb{R}\right\}
$$


where $e_{1}^{2}=-1, e_{2}^{2}=e_{3}^{2}=1$ and $e_{1} e_{2} e_{3}=1$. Real split quaternions are noncommutative, too, [6]. Also, any real split quaternions can be represented by $2 \times 2$ complex matrix, [1]. While coefficients of a real split quaternion are complex numbers, then it is called complex split quaternion. The basis elements of a complex split quaternion have the same rules of a real split quaternion multiplication, [3].

In this study, firstly we associated the results we obtained from the conjugates of the complex quaternion with the real quaternions. Also, we give some properties of matrix representation of complex quaternions and complex split quaternions by expressing these quaternions as $2 \times 2$ complex matrices $\left(M_{2}(\mathbb{C})\right)$ with using matrices corresponding to the basis of complex quaternions and complex split quaternions. Moreover, we obtain a method to find the determinant for these form of quaternions. Finally, we investigate some special matrices for complex quaternion and complex split quaternion matrices.

\section{COMPLEX QUATERNION MATRICES}

A real quaternion $a$ is a vector of the form $a=a_{0} e_{0}+a_{1} e_{1}+a_{2} e_{2}+a_{3} e_{3}$ where $a_{0}, a_{1}, a_{2}, a_{3}$ are real numbers. Here $\left\{e_{0}, e_{1}, e_{2}, e_{3}\right\}$ denotes the set of real quaternion basis with the properties

$$
\begin{gathered}
e_{1}^{2}=e_{2}^{2}=e_{3}^{2}=e_{1} e_{2} e_{3}=-1, \\
e_{1} e_{2}=-e_{2} e_{1}=e_{3}, e_{2} e_{3}=-e_{3} e_{2}=e_{1}, e_{3} e_{1}=-e_{1} e_{3}=e_{2} .
\end{gathered}
$$

A real quaternion $a$ can be written as $a=S_{a}+V_{a}$ where $S_{a}=a_{0} e_{0}$ is the scalar part and $V_{a}=a_{1} e_{1}+a_{2} e_{2}+a_{3} e_{3}$ is the vector part of $a$. For any real quaternion $a=a_{0} e_{0}+a_{1} e_{1}+a_{2} e_{2}+a_{3} e_{3}$, the conjugate of $a$ is $\bar{a}=a_{0} e_{0}-a_{1} e_{1}-a_{2} e_{2}-a_{3} e_{3}$ and the norm of $a$ is $\|a\|=\sqrt{\bar{a} a}=\sqrt{a \bar{a}}=\sqrt{a_{0}^{2}+a_{1}^{2}+a_{2}^{2}+a_{3}^{2}}$. For details, see [2].

A complex quaternion $q$ is of the form $q=A_{0} e_{0}+A_{1} e_{1}+A_{2} e_{2}+A_{3} e_{3}$ where $A_{0}, A_{1}, A_{2}, A_{3}$ are complex numbers and the elements of $\left\{e_{0}, e_{1}, e_{2}, e_{3}\right\}$ multiply as in real quaternions. Also, a complex quaternion $q$ can be written as $\sum\left(a_{k}+i b_{k}\right) e_{k}$ where $a_{k}, b_{k}$ are real numbers for $0 \leq k \leq 3$. Here $i$ denotes the complex unit and commutes with $e_{0}, e_{1}, e_{2}, e_{3}$.

For any complex quaternion $q=A_{0} e_{0}+A_{1} e_{1}+A_{2} e_{2}+A_{3} e_{3}$, the quaternion conjugate of $q$ is $\bar{q}=A_{0} e_{0}-A_{1} e_{1}-A_{2} e_{2}-A_{3} e_{3}$ and $\bar{q} q=q \bar{q}=A_{0}^{2}+A_{1}^{2}+A_{2}^{2}+A_{3}^{2}$. The complex conjugate of $q$ is $q^{c}=\bar{A}_{0} e_{0}+\bar{A}_{1} e_{1}+\bar{A}_{2} e_{2}+\bar{A}_{3} e_{3}$ and the Hermitian conjugate of $q$ is $(\bar{q})^{c}=\bar{A}_{0} e_{0}-\bar{A}_{1} e_{1}-\bar{A}_{2} e_{2}-\bar{A}_{3} e_{3}$. For more information of complex quaternions the reader is referred to [5] and [4]. For a complex quaternion $q=\left(a_{0}+i b_{0}\right) e_{0}+\left(a_{1}+i b_{1}\right) e_{1}+\left(a_{2}+i b_{2}\right) e_{2}+\left(a_{3}+i b_{3}\right) e_{3}$, we express the equalities below related to real quaternions and complex quaternions with using the complex conjugate and the Hermitian conjugate of a complex quaternion.

$$
q^{c} q=a^{2}+b^{2}+2 i\left(V_{a} \times V_{b}\right)
$$




$$
\begin{gathered}
q q^{c}=a^{2}+b^{2}-2 i\left(V_{a} \times V_{b}\right) \\
(\bar{q})^{c} q=\|a\|^{2}+\|b\|^{2}+2 i\left(S_{a} V_{b}-S_{b} V_{a}-V_{a} \times V_{b}\right) \\
q(\bar{q})^{c}=\|a\|^{2}+\|b\|^{2}+2 i\left(S_{a} V_{b}-S_{b} V_{a}+V_{a} \times V_{b}\right)
\end{gathered}
$$

where $a=a_{0} e_{0}+a_{1} e_{1}+a_{2} e_{2}+a_{3} e_{3}, b=b_{0} e_{0}+b_{1} e_{1}+b_{2} e_{2}+b_{3} e_{3}$ and $\times$ denotes the vector product in $\mathbb{R}^{3}$.

A complex quaternion matrix $Q$ is of the form

$$
Q=Q_{0} E_{0}+Q_{1} E_{1}+Q_{2} E_{2}+Q_{3} E_{3}
$$

where $Q_{0}, Q_{1}, Q_{2}, Q_{3}$ are complex numbers. The complex quaternion matrix basis $\left\{E_{0}, E_{1}, E_{2}, E_{3}\right\}$ satisfying the equalities

$$
\begin{gathered}
E_{1}^{2}=E_{2}^{2}=E_{3}^{2}=-E_{0}, \\
E_{1} E_{2}=-E_{2} E_{1}=E_{3}, E_{2} E_{3}=-E_{3} E_{2}=E_{1}, E_{3} E_{1}=-E_{1} E_{3}=E_{2} .
\end{gathered}
$$

These basis elements are $2 \times 2$ matrices, [5]:

$$
E_{0}=\left(\begin{array}{ll}
1 & 0 \\
0 & 1
\end{array}\right), E_{1}=\left(\begin{array}{cc}
i & 0 \\
0 & -i
\end{array}\right), E_{2}=\left(\begin{array}{cc}
0 & 1 \\
-1 & 0
\end{array}\right), E_{3}=\left(\begin{array}{cc}
0 & i \\
i & 0
\end{array}\right) \text {. }
$$

The multiplication rules of the $2 \times 2$ complex matrices $E_{0}, E_{1}, E_{2}, E_{3}$ satisfy the multiplication rules of the complex quaternion basis elements $e_{0}, e_{1}, e_{2}, e_{3}$. Hence, there is an isomorphic relation between the vector form and the matrix form of a complex quaternion.

We denote the algebra of complex quaternion matrices by $\mathbf{H}^{\mathbb{C}}$ and define with the algebra of $2 \times 2$ complex matrices:

$$
\mathbf{H}^{\mathbb{C}}=\left\{Q_{0} E_{0}+Q_{1} E_{1}+Q_{2} E_{2}+Q_{3} E_{3}=\left(\begin{array}{cc}
Q_{0}+i Q_{1} & Q_{2}+i Q_{3} \\
-Q_{2}+i Q_{3} & Q_{0}-i Q_{1}
\end{array}\right): Q_{0}, Q_{1}, Q_{2}, Q_{3} \in \mathbb{C}\right\}
$$

For any $Q=Q_{0} E_{0}+Q_{1} E_{1}+Q_{2} E_{2}+Q_{3} E_{3} \in \mathbf{H}^{\mathbb{C}}$, we define $S_{Q}=Q_{0} E_{0}$, the scalar matrix part of $Q ; \operatorname{Im} Q=Q_{1} E_{1}+Q_{2} E_{2}+Q_{3} E_{3}$, the imaginary matrix part of $Q$. The conjugate, the complex conjugate and the total conjugate of a complex quaternion matrix are denoted by $\bar{Q}, Q^{C},(\bar{Q})^{C}$ respectively these are

$$
\begin{aligned}
\bar{Q} & =Q_{0} E_{0}-Q_{1} E_{1}-Q_{2} E_{2}-Q_{3} E_{3}, \\
Q^{C} & =Q_{0} \bar{E}_{0}+Q_{1} \bar{E}_{1}+Q_{2} \bar{E}_{2}+Q_{3} \bar{E}_{3} \\
& =Q_{0} E_{0}-Q_{1} E_{1}+Q_{2} E_{2}-Q_{3} E_{3},
\end{aligned}
$$




$$
\begin{aligned}
(\bar{Q})^{C} & =\overline{\left(Q^{C}\right)}=Q_{0} \bar{E}_{0}-Q_{1} \bar{E}_{1}-Q_{2} \bar{E}_{2}-Q_{3} \bar{E}_{3} \\
& =Q_{0} E_{0}+Q_{1} E_{1}-Q_{2} E_{2}+Q_{3} E_{3} .
\end{aligned}
$$

In addition, for any $Q=Q_{0} E_{0}+Q_{1} E_{1}+Q_{2} E_{2}+Q_{3} E_{3} \in \mathbf{H}^{\mathbb{C}}$, we can define transpose and adjoint matrix of $Q$ by $Q^{t}$ and $A d j Q$ respectively write down as

$$
\begin{gathered}
Q^{t}=Q_{0} E_{0}+Q_{1} E_{1}-Q_{2} E_{2}+Q_{3} E_{3}, \\
\operatorname{Adj} Q=Q_{0} E_{0}-Q_{1} E_{1}-Q_{2} E_{2}-Q_{3} E_{3} .
\end{gathered}
$$

So we can get

$$
\begin{aligned}
& \operatorname{Adj} Q=\bar{Q}, \\
& Q^{C}=(\bar{Q})^{t} .
\end{aligned}
$$

The norm of a complex quaternion matrix

$$
Q=Q_{0} E_{0}+Q_{1} E_{1}+Q_{2} E_{2}+Q_{3} E_{3}=\left(\begin{array}{cc}
q_{11} & q_{12} \\
-\bar{q}_{12} & \bar{q}_{11}
\end{array}\right)
$$

is defined as

$$
\|Q\|=\sqrt{\left.|| q_{11}\right|^{2}+\left|q_{12}\right|^{2} \mid}
$$

where $q_{11}=Q_{0}+i Q_{1}$ and $q_{12}=Q_{2}+i Q_{3}$.

Definition 1. A determinant of $Q \in \mathbf{H}^{\mathbb{C}}$ is defined as

$$
\operatorname{det} Q=Q_{0}^{2} \operatorname{det} E_{0}+Q_{1}^{2} \operatorname{det} E_{1}+Q_{2}^{2} \operatorname{det} E_{2}+Q_{3}^{2} \operatorname{det} E_{3} \text {. }
$$

Using the determinant of a complex quaternion matrix basis the above determinant can be written as

$$
\operatorname{det} Q=Q_{0}^{2}+Q_{1}^{2}+Q_{2}^{2}+Q_{3}^{2} .
$$

Theorem 1. For any $Q, P \in \mathbf{H}^{\mathbb{C}}$ and $\lambda \in \mathbb{C}$ the following properties are satisfied: (i) $\operatorname{det} Q=\operatorname{det}(\bar{Q})=\operatorname{det}\left(Q^{C}\right)=\operatorname{det}\left(Q^{t}\right)$,

(ii) $\operatorname{det}(\lambda Q)=\lambda^{2} \operatorname{det} Q$,

(iii) $\operatorname{det}(Q P)=\operatorname{det} Q \operatorname{det} P$.

Proof. (i) For $Q=Q_{0} E_{0}+Q_{1} E_{1}+Q_{2} E_{2}+Q_{3} E_{3} \in \mathbf{H}^{\mathbb{C}}$, from (2.22) it can be found easily that

$$
\operatorname{det} Q=\operatorname{det}(\bar{Q})=\operatorname{det}\left(Q^{C}\right)=\operatorname{det}\left(Q^{t}\right)=Q_{0}^{2}+Q_{1}^{2}+Q_{2}^{2}+Q_{3}^{2} .
$$

(ii) For any $\lambda \in \mathbb{C}$, we have $\lambda Q=\left(\lambda Q_{0}\right) E_{0}+\left(\lambda Q_{1}\right) E_{1}+\left(\lambda Q_{2}\right) E_{2}+\left(\lambda Q_{3}\right) E_{3}$. Thus,

$$
\begin{aligned}
\operatorname{det}(\lambda Q) & =\lambda^{2} Q_{0}^{2}+\lambda^{2} Q_{1}^{2}+\lambda^{2} Q_{2}^{2}+\lambda^{2} Q_{3}^{2} \\
& =\lambda^{2}\left(Q_{0}^{2}+Q_{1}^{2}+Q_{2}^{2}+Q_{3}^{2}\right)
\end{aligned}
$$




$$
=\lambda^{2} \operatorname{det} Q \text {. }
$$

(iii) Let $Q=Q_{0} E_{0}+Q_{1} E_{1}+Q_{2} E_{2}+Q_{3} E_{3}$ and $P=P_{0} E_{0}+P_{1} E_{1}+P_{2} E_{2}+P_{3} E_{3}$ be complex quaternion matrices, $Q P$ is calculated as

$$
\begin{aligned}
Q P & =\left(Q_{0} P_{0}-Q_{1} P_{1}-Q_{2} P_{2}-Q_{3} P_{3}\right) E_{0}+\left(Q_{0} P_{1}+Q_{1} P_{0}+Q_{2} P_{3}-Q_{3} P_{2}\right) E_{1} \\
& +\left(Q_{0} P_{2}+Q_{2} P_{0}-Q_{1} P_{3}+Q_{3} P_{1}\right) E_{2}+\left(Q_{0} P_{3}+Q_{3} P_{0}+Q_{1} P_{2}-Q_{2} P_{1}\right) E_{3}
\end{aligned}
$$

and from (2.22) we have

$$
\begin{aligned}
\operatorname{det}(Q P) & =Q_{0}^{2} P_{0}^{2}+Q_{0}^{2} P_{1}^{2}+Q_{0}^{2} P_{2}^{2}+Q_{0}^{2} P_{3}^{2}+Q_{1}^{2} P_{0}^{2}+Q_{1}^{2} P_{1}^{2}+Q_{1}^{2} P_{2}^{2}+Q_{1}^{2} P_{3}^{2} \\
& +Q_{2}^{2} P_{0}^{2}+Q_{2}^{2} P_{1}^{2}+Q_{2}^{2} P_{2}^{2}+Q_{2}^{2} P_{3}^{2}+Q_{3}^{2} P_{0}^{2}+Q_{3}^{2} P_{1}^{2}+Q_{3}^{2} P_{2}^{2}+Q_{3}^{2} P_{3}^{2} .
\end{aligned}
$$

On the other hand, the determinants of $Q$ and $P$ are $Q_{0}^{2}+Q_{1}^{2}+Q_{2}^{2}+Q_{3}^{2}$ and $P_{0}^{2}+$ $P_{1}^{2}+P_{2}^{2}+P_{3}^{2}$, respectively, then

$\operatorname{det} Q \operatorname{det} P$

$$
\begin{aligned}
& =Q_{0}^{2} P_{0}^{2}+Q_{0}^{2} P_{1}^{2}+Q_{0}^{2} P_{2}^{2}+Q_{0}^{2} P_{3}^{2}+Q_{1}^{2} P_{0}^{2}+Q_{1}^{2} P_{1}^{2}+Q_{1}^{2} P_{2}^{2}+Q_{1}^{2} P_{3}^{2} \\
& +Q_{2}^{2} P_{0}^{2}+Q_{2}^{2} P_{1}^{2}+Q_{2}^{2} P_{2}^{2}+Q_{2}^{2} P_{3}^{2}+Q_{3}^{2} P_{0}^{2}+Q_{3}^{2} P_{1}^{2}+Q_{3}^{2} P_{2}^{2}+Q_{3}^{2} P_{3}^{2} .
\end{aligned}
$$

Therefore

$$
\operatorname{det}(Q P)=\operatorname{det} Q \operatorname{det} P \text {. }
$$

Additionally, using the complex conjugate and the transpose of a complex quaternion matrix we obtain the determinant of a complex quaternion matrix.

$$
Q^{t} Q^{C}=Q^{C} Q^{t}=\left(Q_{0}^{2}+Q_{1}^{2}+Q_{2}^{2}+Q_{3}^{2}\right) E_{0}
$$

and from (2.22) the determinant of $Q^{t} Q^{C}$ is

$$
\operatorname{det}\left(Q^{t} Q^{C}\right)=\left(Q_{0}^{2}+Q_{1}^{2}+Q_{2}^{2}+Q_{3}^{2}\right)^{2}
$$

so,

$$
(\operatorname{det} Q)^{2}=\operatorname{det}\left(Q^{t} Q^{C}\right) \text {. }
$$

If $\operatorname{det} Q \neq 0$, the inverse of a complex quaternion matrix is defined as

$$
Q^{-1}=\frac{1}{\operatorname{det} Q} \bar{Q}
$$

From (2.12), (2.22) and (2.26) the inverse of a complex quaternion matrix can be written as

$$
Q^{-1}=\frac{1}{Q_{0}^{2}+Q_{1}^{2}+Q_{2}^{2}+Q_{3}^{2}}\left(Q_{0} E_{0}-Q_{1} E_{1}-Q_{2} E_{2}-Q_{3} E_{3}\right) .
$$


Example 1. Let $Q=E_{0}+i E_{2}+E_{3}$ be a complex quaternion matrix. Then, the complex quaternion matrix $Q$ can be written as $Q=\left(\begin{array}{cc}1 & 2 i \\ 0 & 1\end{array}\right)$. From (2.27), the inverse of $Q$ is

$$
\begin{aligned}
Q^{-1} & =E_{0}-i E_{2}-E_{3} \\
& =\left(\begin{array}{cc}
1 & -2 i \\
0 & 1
\end{array}\right) .
\end{aligned}
$$

Theorem 2. Complex quaternion matrices satisfy the following properties for $Q \in \mathbf{H}^{\mathbb{C}}$ :

(i) $E_{1} c=c E_{1}, E_{2} c=c E_{2}, E_{3} c=c E_{3}$ for any complex number $c$,

(ii) $Q^{2}=S_{Q}^{2}-\operatorname{det}(\operatorname{Im} Q) E_{0}+2 S_{Q} \operatorname{Im} Q$,

(iii) Every complex quaternion matrix $Q$ is expressed as $Q=Z_{1}+Z_{2} E_{2}$ where $Z_{1}, Z_{2} \in M_{2}(\mathbb{C})$.

Proof. Proofs of ( $i$ ) and (iii) can be easily shown. Now, we will prove (ii). For $Q=Q_{0} E_{0}+Q_{1} E_{1}+Q_{2} E_{2}+Q_{3} E_{3} \in \mathbf{H}^{\mathbb{C}}$,

$$
Q^{2}=\left(Q_{0}^{2}-Q_{1}^{2}-Q_{2}^{2}-Q_{3}^{2}\right) E_{0}+2 Q_{0}\left(Q_{1} E_{1}+Q_{2} E_{2}+Q_{3} E_{3}\right)
$$

and using the following equalities

$$
S_{Q}=Q_{0} E_{0}, \operatorname{Im} Q=Q_{1} E_{1}+Q_{2} E_{2}+Q_{3} E_{3}, \operatorname{det}(\operatorname{Im} Q)=Q_{1}^{2}+Q_{2}^{2}+Q_{3}^{2}
$$

we get

$$
Q^{2}=S_{Q}^{2}-\operatorname{det}(\operatorname{Im} Q) E_{0}+2 S_{Q} \operatorname{Im} Q
$$

Theorem 3. For any $Q, P \in \mathbf{H}^{\mathbb{C}}$ the following properties are satisfied:

(i) $Q=\left[(\bar{Q})^{t}\right]^{C}$,

(ii) $Q^{t}=\overline{\left(Q^{C}\right)}$,

(iii) $\left(Q^{C}\right)^{-1}=\left(Q^{-1}\right)^{C}$ if $Q$ is invertible,

(iv) $(\bar{Q})^{-1}=\overline{\left(Q^{-1}\right)}$ if $Q$ is invertible,

(v) $\left(Q^{t}\right)^{-1}=\left(Q^{-1}\right)^{t}$ if $Q$ is invertible,

(vi) $(Q P)^{C}=Q^{C} P^{C}$,

(vii) $(Q P)^{-1}=P^{-1} Q^{-1}$ if $Q$ and $P$ are invertible.

Proof. Proof of the theorem is easily shown. However, we will prove only (ii), (iii) and (vii).

(ii) For $Q=Q_{0} E_{0}+Q_{1} E_{1}+Q_{2} E_{2}+Q_{3} E_{3} \in \mathbf{H}^{\mathbb{C}}$, the complex conjugate of a 
complex quaternion matrix is $Q^{C}=Q_{0} E_{0}-Q_{1} E_{1}+Q_{2} E_{2}-Q_{3} E_{3}$ and from (2.12) it is obtained that

$$
\overline{\left(Q^{C}\right)}=Q_{0} E_{0}+Q_{1} E_{1}-Q_{2} E_{2}+Q_{3} E_{3} .
$$

The equality (2.15) implies that the transpose of $Q \in \mathbf{H}^{\mathbb{C}}$ is

$$
Q^{t}=Q_{0} E_{0}+Q_{1} E_{1}-Q_{2} E_{2}+Q_{3} E_{3} .
$$

Thus,

$$
Q^{t}=\overline{\left(Q^{C}\right)} \text {. }
$$

(i i i ) Let $Q=Q_{0} E_{0}+Q_{1} E_{1}+Q_{2} E_{2}+Q_{3} E_{3} \in \mathbf{H}^{\mathbb{C}}$ and $Q$ be an invertible complex quaternion matrix. We know $Q^{C}=Q_{0} E_{0}-Q_{1} E_{1}+Q_{2} E_{2}-Q_{3} E_{3}$. From (2.27) we get

$$
\left(Q^{C}\right)^{-1}=\frac{1}{Q_{0}^{2}+Q_{1}^{2}+Q_{2}^{2}+Q_{3}^{2}}\left(Q_{0} E_{0}+Q_{1} E_{1}-Q_{2} E_{2}+Q_{3} E_{3}\right) .
$$

From (2.27) and (2.13), we find

$$
\left(Q^{-1}\right)^{C}=\frac{1}{Q_{0}^{2}+Q_{1}^{2}+Q_{2}^{2}+Q_{3}^{2}}\left(Q_{0} E_{0}+Q_{1} E_{1}-Q_{2} E_{2}+Q_{3} E_{3}\right) .
$$

So,

$$
\left(Q^{C}\right)^{-1}=\left(Q^{-1}\right)^{C} \text {. }
$$

(vi i) Let $Q=Q_{0} E_{0}+Q_{1} E_{1}+Q_{2} E_{2}+Q_{3} E_{3}$ and

$P=P_{0} E_{0}+P_{1} E_{1}+P_{2} E_{2}+P_{3} E_{3}$ be invertible complex quaternion matrices. We denote $Q P$ as

$$
Q P=A E_{0}+B E_{1}+C E_{2}+D E_{3},
$$

for simplicity, where

$$
\begin{aligned}
& A=Q_{0} P_{0}-Q_{1} P_{1}-Q_{2} P_{2}-Q_{3} P_{3}, B=Q_{0} P_{1}+Q_{1} P_{0}+Q_{2} P_{3}-Q_{3} P_{2}, \\
& C=Q_{0} P_{2}-Q_{1} P_{3}+Q_{2} P_{0}+Q_{3} P_{1}, D=Q_{0} P_{3}+Q_{1} P_{2}-Q_{2} P_{1}+Q_{3} P_{0}
\end{aligned}
$$

From (2.22) the determinant of $Q P$ is written as

$$
\operatorname{det}(Q P)=A^{2}+B^{2}+C^{2}+D^{2},
$$

where

$$
\begin{aligned}
& A^{2}+B^{2}+C^{2}+D^{2} \\
& =Q_{0}^{2} P_{0}^{2}+Q_{0}^{2} P_{1}^{2}+Q_{0}^{2} P_{2}^{2}+Q_{0}^{2} P_{3}^{2}+Q_{1}^{2} P_{0}^{2}+Q_{1}^{2} P_{1}^{2}+Q_{1}^{2} P_{2}^{2}+Q_{1}^{2} P_{3}^{2} \\
& \quad+Q_{2}^{2} P_{0}^{2}+Q_{2}^{2} P_{1}^{2}+Q_{2}^{2} P_{2}^{2}+Q_{2}^{2} P_{3}^{2}+Q_{3}^{2} P_{0}^{2}+Q_{3}^{2} P_{1}^{2}+Q_{3}^{2} P_{2}^{2}+Q_{3}^{2} P_{3}^{2}
\end{aligned}
$$


and from (2.27) can be found

$$
(Q P)^{-1}=\frac{\overline{(Q P)}}{\operatorname{det}(Q P)}=\frac{A E_{0}-B E_{1}-C E_{2}-D E_{3}}{A^{2}+B^{2}+C^{2}+D^{2}} .
$$

On the other hand, from (2.27), the inverses of $P$ and $Q$ can be written as

$$
P^{-1}=\frac{P_{0} E_{0}-P_{1} E_{1}-P_{2} E_{2}-P_{3} E_{3}}{P_{0}^{2}+P_{1}^{2}+P_{2}^{2}+P_{3}^{2}} \text { and } Q^{-1}=\frac{Q_{0} E_{0}-Q_{1} E_{1}-Q_{2} E_{2}-Q_{3} E_{3}}{Q_{0}^{2}+Q_{1}^{2}+Q_{2}^{2}+Q_{3}^{2}}
$$

and their product is obtained as

Therefore,

$$
P^{-1} Q^{-1}=\frac{A E_{0}-B E_{1}-C E_{2}-D E_{3}}{A^{2}+B^{2}+C^{2}+D^{2}} .
$$

$$
(Q P)^{-1}=P^{-1} Q^{-1}
$$

Example 2. Let $Q=E_{0}+E_{1}, P=E_{0}+E_{2} \in \mathbf{H}^{\mathbb{C}}$. Then,

(i) $(Q P)^{C}=E_{0}-E_{1}+E_{2}-E_{3} \neq E_{0}-E_{1}+E_{2}+E_{3}=P^{C} Q^{C}$

(ii) $(Q P)^{-1}=\frac{1}{4}\left(E_{0}-E_{1}-E_{2}-E_{3}\right) \neq \frac{1}{4}\left(E_{0}-E_{1}-E_{2}+E_{3}\right)=Q^{-1} P^{-1}$

Example 3. Let $Q=E_{0}+E_{1}+E_{2}$. Then,

$Q(\bar{Q})^{C}=E_{0}+2 E_{1}-2 E_{3} \neq E_{0}+2 E_{1}+2 E_{3}=(\bar{Q})^{C} Q$.

With these examples we get the following Corollary for complex quaternion matrices.

Corollary 1. Let $Q, P \in \mathbf{H}^{\mathbb{C}}$. Then the followings are satisfied:

(i) $(Q P)^{C} \neq P^{C} Q^{C}$ in general;

(ii) $(Q P)^{-1} \neq Q^{-1} P^{-1}$ in general;

(iii) $Q(\bar{Q})^{C} \neq(\bar{Q})^{C} Q$ in general.

Definition 2. For any $Q=Q_{0} E_{0}+Q_{1} E_{1}+Q_{2} E_{2}+Q_{3} E_{3} \in \mathbf{H}^{\mathbb{C}}$,

(i) if off-diagonal entries of $Q$ are 0 then $Q$ is called a diagonal matrix and $Q$ is in form of $Q=Q_{0} E_{0}+Q_{1} E_{1}$,

(ii) if $Q^{t}=Q$ then $Q$ is called a symmetric matrix and $Q$ is in form of $Q=Q_{0} E_{0}+$ $Q_{1} E_{1}+Q_{3} E_{3}$,

(iii) if $Q^{t}=Q^{-1}$ then $Q$ is called a orthogonal matrix and $Q$ is in form of $Q=$ $Q_{0} E_{0}+Q_{2} E_{2}$ and $\operatorname{det} Q=1$,

(iv) if $(\bar{Q})^{t}=Q$ then $Q$ is called a Hermitian matrix and $Q$ is in form of $Q=$ $Q_{0} E_{0}+Q_{2} E_{2}$,

(v) if $(\bar{Q})^{t}=Q^{-1}$ then $Q$ is called a unitary matrix and $Q$ is in form of $Q=$ $Q_{0} E_{0}+Q_{1} E_{1}+Q_{3} E_{3}$ and $\operatorname{det} Q=1$. 


\section{COMPLEX SPLIT QUATERNION MATRICES}

A complex split quaternion $p$ is a vector of the form $p=b_{0} e_{0}+b_{1} e_{1}+b_{2} e_{2}+$ $b_{3} e_{3}$ where $b_{0}, b_{1}, b_{2}, b_{3}$ are complex numbers. Here $\left\{e_{0}, e_{1}, e_{2}, e_{3}\right\}$ denotes the complex split quaternion basis with the below properties

$$
\begin{gathered}
e_{1}^{2}=-1, e_{2}^{2}=e_{3}^{2}=e_{1} e_{2} e_{3}=1, \\
e_{1} e_{2}=-e_{2} e_{1}=e_{3}, e_{2} e_{3}=-e_{3} e_{2}=-e_{1}, e_{3} e_{1}=-e_{1} e_{3}=e_{2} .
\end{gathered}
$$

For details of complex split quaternions, see [3].

A complex split quaternion matrix $P$ is of the form

$$
P=P_{0} E_{0}+P_{1} E_{1}+P_{2} E_{2}+P_{3} E_{3}
$$

where $P_{0}, P_{1}, P_{2}, P_{3}$ are complex numbers. The split quaternion matrix basis $\left\{E_{0}, E_{1}, E_{2}, E_{3}\right\}$ satisfy the equalities

$$
\begin{gathered}
E_{1}^{2}=-E_{0}, E_{2}^{2}=E_{3}^{2}=E_{0}, \\
E_{1} E_{2}=-E_{2} E_{1}=E_{3}, E_{2} E_{3}=-E_{3} E_{2}=-E_{1}, E_{3} E_{1}=-E_{1} E_{3}=E_{2} .
\end{gathered}
$$

These basis elements are $2 \times 2$ matrices, [6]:

$$
E_{0}=\left(\begin{array}{ll}
1 & 0 \\
0 & 1
\end{array}\right), E_{1}=\left(\begin{array}{cc}
i & 0 \\
0 & -i
\end{array}\right), E_{2}=\left(\begin{array}{ll}
0 & 1 \\
1 & 0
\end{array}\right), E_{3}=\left(\begin{array}{cc}
0 & i \\
-i & 0
\end{array}\right) .
$$

The multiplication rules of the complex $2 \times 2$ matrices $E_{0}, E_{1}, E_{2}, E_{3}$ coincide with the multiplication rules of the complex split quaternion basis elements $e_{0}, e_{1}, e_{2}, e_{3}$. Hence, there is an isomorphic relation between the vector form and the matrix form of a complex split quaternion.

Let us denote the algebra of complex split quaternion matrices by $\mathbf{H}_{\mathrm{S}}^{\mathbb{C}} \cdot \mathbf{H}_{\mathrm{S}}^{\mathbb{C}}$ can be defined with the algebra of $2 \times 2$ complex matrices:

$$
\mathbf{H}_{\mathbf{S}}^{\mathbb{C}}=\left\{P_{0} E_{0}+P_{1} E_{1}+P_{2} E_{2}+P_{3} E_{3}=\left(\begin{array}{cc}
P_{0}+i P_{1} & P_{2}+i P_{3} \\
P_{2}-i P_{3} & P_{0}-i P_{1}
\end{array}\right): P_{0}, P_{1}, P_{2}, P_{3} \in \mathbb{C}\right\}
$$

For any $P=P_{0} E_{0}+P_{1} E_{1}+P_{2} E_{2}+P_{3} E_{3} \in \mathbf{H}_{\mathbf{S}}^{\mathbb{C}}$, we define $S_{P}=P_{0} E_{0}$, the scalar matrix part of $P ; \operatorname{Im} P=P_{1} E_{1}+P_{2} E_{2}+P_{3} E_{3}$, the imaginary matrix part of $P$. The conjugate, the complex conjugate and the total conjugate of a complex split quaternion matrix are denoted by $\bar{P}, P^{C},(\bar{P})^{C}$ respectively these are

$$
\begin{gathered}
\bar{P}=P_{0} E_{0}-P_{1} E_{1}-P_{2} E_{2}-P_{3} E_{3}, \\
P^{C}=P_{0} \bar{E}_{0}+P_{1} \bar{E}_{1}+P_{2} \bar{E}_{2}+P_{3} \bar{E}_{3} \\
=P_{0} E_{0}-P_{1} E_{1}+P_{2} E_{2}-P_{3} E_{3}, \\
(\bar{P})^{C}=\overline{\left(P^{C}\right)}=P_{0} \bar{E}_{0}-P_{1} \bar{E}_{1}-P_{2} \bar{E}_{2}-P_{3} \bar{E}_{3}
\end{gathered}
$$




$$
=P_{0} E_{0}+P_{1} E_{1}-P_{2} E_{2}+P_{3} E_{3} .
$$

Moreover, for any $P=P_{0} E_{0}+P_{1} E_{1}+P_{2} E_{2}+P_{3} E_{3} \in \mathbf{H}_{\mathrm{S}}^{\mathbb{C}}$, we can define transpose and adjoint matrix of $P$ by $P^{t}$ and $\operatorname{Adj} P$ and these are

$$
\begin{gathered}
P^{t}=P_{0} E_{0}+P_{1} E_{1}+P_{2} E_{2}-P_{3} E_{3}, \\
A d j P=P_{0} E_{0}-P_{1} E_{1}-P_{2} E_{2}-P_{3} E_{3}, \\
A d j P=\bar{P} .
\end{gathered}
$$

The norm of a complex split quaternion matrix

$$
P=P_{0} E_{0}+P_{1} E_{1}+P_{2} E_{2}+P_{3} E_{3}=\left(\begin{array}{cc}
p_{11} & p_{12} \\
\bar{p}_{12} & \bar{p}_{11}
\end{array}\right)
$$

is defined as

$$
\|P\|=\sqrt{\left.|| p_{11}\right|^{2}-\left|p_{12}\right|^{2} \mid}
$$

where $p_{11}=P_{0}+i P_{1}$ and $p_{12}=P_{2}+i P_{3}$.

Definition 3. A determinant of $P \in \mathbf{H}_{\mathrm{S}}^{\mathbb{C}}$ is defined as

$$
\operatorname{det} P=P_{0}^{2} \operatorname{det} E_{0}+P_{1}^{2} \operatorname{det} E_{1}+P_{2}^{2} \operatorname{det} E_{2}+P_{3}^{2} \operatorname{det} E_{3} .
$$

From the determinant of a complex split quaternion basis can be written as

$$
\operatorname{det} P=P_{0}^{2}+P_{1}^{2}-P_{2}^{2}-P_{3}^{2} \text {. }
$$

Theorem 4. For any $P, Q \in \mathbf{H}_{\mathbf{S}}^{\mathbb{C}}$ and $\psi \in \mathbb{C}$ the following properties are satisfied: (i) $\operatorname{det} P=\operatorname{det}(\bar{P})=\operatorname{det}\left(P^{C}\right)=\operatorname{det}\left(P^{t}\right)$,

(ii) $\operatorname{det}(\psi P)=\psi^{2} \operatorname{det} P$,

(iii) $\operatorname{det}(P Q)=\operatorname{det} P \operatorname{det} Q$.

Proof. (i) For $P=P_{0} E_{0}+P_{1} E_{1}+P_{2} E_{2}+P_{3} E_{3} \in \mathbf{H}_{\mathbf{S}}^{\mathbb{C}}$, from (3.17) we get

$$
\operatorname{det} P=\operatorname{det}(\bar{P})=\operatorname{det}\left(P^{C}\right)=\operatorname{det}\left(P^{t}\right)=P_{0}^{2}+P_{1}^{2}-P_{2}^{2}-P_{3}^{2} \text {. }
$$

(ii) For any $\psi \in \mathbb{C}$ and $P=P_{0} E_{0}+P_{1} E_{1}+P_{2} E_{2}+P_{3} E_{3} \in \mathbf{H}_{\mathbf{S}}^{\mathbb{C}}$,

$$
\psi P=\left(\psi P_{0}\right) E_{0}+\left(\psi P_{1}\right) E_{1}+\left(\psi P_{2}\right) E_{2}+\left(\psi P_{3}\right) E_{3} \text {. }
$$

Thus,

$$
\begin{aligned}
\operatorname{det}(\psi P) & =\psi^{2} P_{0}^{2}+\psi^{2} P_{1}^{2}-\psi^{2} P_{2}^{2}-\psi^{2} P_{3}^{2} \\
& =\psi^{2}\left(P_{0}^{2}+P_{1}^{2}-P_{2}^{2}-P_{3}^{2}\right) \\
& =\psi^{2} \operatorname{det} P .
\end{aligned}
$$

(iii) Let $P=P_{0} E_{0}+P_{1} E_{1}+P_{2} E_{2}+P_{3} E_{3}$ and $Q=Q_{0} E_{0}+Q_{1} E_{1}+Q_{2} E_{2}+Q_{3} E_{3}$ be complex split quaternion matrices, then $P Q$ 


$$
\begin{aligned}
= & \left(P_{0} Q_{0}-P_{1} Q_{1}+P_{2} Q_{2}+P_{3} Q_{3}\right) E_{0}+\left(P_{0} Q_{1}+P_{1} Q_{0}-P_{2} Q_{3}+P_{3} Q_{2}\right) E_{1} \\
& +\left(P_{0} Q_{2}+P_{2} Q_{0}-P_{1} Q_{3}+P_{3} Q_{1}\right) E_{2}+\left(P_{0} Q_{3}+P_{3} Q_{0}+P_{1} Q_{2}-P_{2} Q_{1}\right) E_{3}
\end{aligned}
$$

and from (3.17) the determinant of $P Q$ can be found as in the form of

$$
\begin{aligned}
& \operatorname{det}(P Q) \\
& =P_{0}^{2} Q_{0}^{2}+P_{0}^{2} Q_{1}^{2}+P_{1}^{2} Q_{0}^{2}+P_{1}^{2} Q_{1}^{2}+P_{2}^{2} Q_{2}^{2}+P_{2}^{2} Q_{3}^{2}+P_{3}^{2} Q_{2}^{2}+P_{3}^{2} Q_{3}^{2} \\
& \quad-P_{0}^{2} Q_{2}^{2}-P_{0}^{2} Q_{3}^{2}-P_{1}^{2} Q_{2}^{2}-P_{1}^{2} Q_{3}^{2}-P_{2}^{2} Q_{0}^{2}-P_{2}^{2} Q_{1}^{2}-P_{3}^{2} Q_{0}^{2}-P_{3}^{2} Q_{1}^{2} .
\end{aligned}
$$

On the other hand, the determinants of $P$ and $Q$ are $P_{0}^{2}+P_{1}^{2}-P_{2}^{2}-P_{3}^{2}$ and $Q_{0}^{2}+$ $Q_{1}^{2}-Q_{2}^{2}-Q_{3}^{2}$ respectively

$$
\begin{aligned}
& \operatorname{det} P \operatorname{det} Q \\
& =P_{0}^{2} Q_{0}^{2}+P_{0}^{2} Q_{1}^{2}+P_{1}^{2} Q_{0}^{2}+P_{1}^{2} Q_{1}^{2}+P_{2}^{2} Q_{2}^{2}+P_{2}^{2} Q_{3}^{2}+P_{3}^{2} Q_{2}^{2}+P_{3}^{2} Q_{3}^{2} \\
& \quad-P_{0}^{2} Q_{2}^{2}-P_{0}^{2} Q_{3}^{2}-P_{1}^{2} Q_{2}^{2}-P_{1}^{2} Q_{3}^{2}-P_{2}^{2} Q_{0}^{2}-P_{2}^{2} Q_{1}^{2}-P_{3}^{2} Q_{0}^{2}-P_{3}^{2} Q_{1}^{2} .
\end{aligned}
$$

Thus,

$$
\operatorname{det}(P Q)=\operatorname{det} P \operatorname{det} Q
$$

Moreover, the determinant of a complex split quaternion matrix can also be found by the complex conjugate and the total conjugate of a complex split quaternion matrix.

$$
{ }^{C}(\bar{P})^{C}=(\bar{P})^{C} P^{C}=\left(P_{0}^{2}+P_{1}^{2}-P_{2}^{2}-P_{3}^{2}\right) E_{0}
$$

and from (3.17) the determinant of $P^{C}(\bar{P})^{C}$ is found as

$$
\operatorname{det}\left(P^{C}(\bar{P})^{C}\right)=\left(P_{0}^{2}+P_{1}^{2}-P_{2}^{2}-P_{3}^{2}\right)^{2} .
$$

Hence, the determinant of a complex split quaternion matrix can be written as

$$
(\operatorname{det} P)^{2}=\operatorname{det}\left(P^{C}(\bar{P})^{C}\right) .
$$

If $\operatorname{det} P \neq 0$, the inverse of a complex split quaternion matrix is defined as

$$
P^{-1}=\frac{1}{\operatorname{det} P} \bar{P}
$$

From (3.8), (3.17) and (3.21) can be written

$$
Q^{-1}=\frac{1}{P_{0}^{2}+P_{1}^{2}-P_{2}^{2}-P_{3}^{2}}\left(P_{0} E_{0}-P_{1} E_{1}-P_{2} E_{2}-P_{3} E_{3}\right) .
$$


Example 4. Let $P=\left(\frac{1-i}{2}\right) E_{0}+\left(\frac{1-i}{2}\right) E_{1}-\frac{1}{2} E_{2}+\frac{i}{2} E_{3}$ be a complex split quaternion matrix. Then, $P$ can be written as $P=\left(\begin{array}{ll}1 & -1 \\ 0 & -i\end{array}\right)$ and from (3.22), the inverse of $P$ is calculated as

$$
\begin{aligned}
P^{-1} & =\left(\frac{1+i}{2}\right) E_{0}+\left(\frac{-1-i}{2}\right) E_{1}+\frac{i}{2} E_{2}+\frac{1}{2} E_{3} \\
& =\left(\begin{array}{cc}
1 & i \\
0 & i
\end{array}\right) .
\end{aligned}
$$

Theorem 5. Complex split quaternion matrices satisfy the following properties for $P \in \mathbf{H}_{\mathrm{S}}^{\mathbb{C}}$.

(i) $E_{1} c=c E_{1}, E_{2} c=c E_{2}, E_{3} c=c E_{3}$ for any complex number $c$,

(ii) $P^{2}=S_{P}^{2}-\operatorname{det}(\operatorname{Im} P) E_{0}+2 S_{P} \operatorname{Im} P$,

(iii) Every complex split quaternion matrix $P$ can be uniquely expressed as $P=$ $Z_{1}+Z_{2} E_{2}$, where $Z_{1}, Z_{2} \in M_{2}(\mathbb{C})$.

Theorem 6. For any $P, Q \in \mathbf{H}_{\mathbf{S}}^{\mathbb{C}}$ the following properties are satisfied:

(i) $\operatorname{det} P=\|P\|^{2}$,

(ii ) $(\bar{P})^{-1}=\overline{\left(P^{-1}\right)}$ if $P$ is invertible,

(ii i ) $\left(P^{C}\right)^{-1}=\left(P^{-1}\right)^{C}$ if $P$ is invertible,

(iv) $\left[(\bar{P})^{t}\right]^{-1}=\left[\overline{\left(P^{-1}\right)}\right]^{t}$ if $P$ is invertible,

(v) $(P Q)^{C}=P^{C} Q^{C}$,

(vi) $(P Q)^{-1}=Q^{-1} P^{-1}$ if $P$ and $Q$ are invertible.

The proof is analogous to the proof of Theorem 3.

Example 5. Let $P=E_{0}+E_{2}+E_{3}$ and $Q=E_{0}+E_{1}$. Then,

(i) $(P Q)^{C}=E_{0}-E_{1}+2 E_{2} \neq E_{0}-E_{1}-2 E_{3}=Q^{C} P^{C}$,

(ii) $P(\bar{P})^{C}=E_{0}-2 E_{1}+2 E_{3} \neq E_{0}+2 E_{1}+2 E_{3}=(\bar{P})^{C} P$.

With these examples we get the following Corollary for complex split quaternion matrices.

Corollary 2. Let $P, Q \in \mathbf{H}_{\mathrm{S}}^{\mathbb{C}}$. Then the followings are satisfied:

(i) $(P Q)^{C} \neq Q^{C} P^{C}$ in general;

(i i ) $P(\bar{P})^{C} \neq(\bar{P})^{C} P$ in general.

Definition 4. For any $P=P_{0} E_{0}+P_{1} E_{1}+P_{2} E_{2}+P_{3} E_{3} \in \mathbf{H}_{\mathbf{S}}^{\mathbb{C}}$,

(i) if off-diagonal entries of $P$ are 0 then $P$ is called a diagonal matrix and $P$ is in 
form of $P_{0} E_{0}+P_{1} E_{1}$,

(ii) if $P^{t}=P$ then $P$ is called a symmetric matrix and $P$ is in form of $P=$ $P_{0} E_{0}+P_{1} E_{1}+P_{2} E_{2}$,

(iii) if $P^{t}=P^{-1}$ then $P$ is called an orthogonal matrix and $P$ is in form of $P=P_{0} E_{0}+P_{3} E_{3}$ and $\operatorname{det} P=1$,

(iv) if $(\bar{P})^{t}=P$ then $P$ is called a Hermitian matrix and $P$ is in form of $P=$ $P_{0} E_{0}+P_{3} E_{3}$,

(v) if $(\bar{P})^{t}=P^{-1}$ then $P$ is called an unitary matrix and $P$ is in form of $P=$ $P_{0} E_{0}+P_{1} E_{1}+P_{2} E_{2}$ and $\operatorname{det} P=1$.

\section{CONCLUSion}

This paper has investigated the main properties of complex quaternion and complex split quaternion matrices with the use of $2 \times 2$ complex matrix representation of them, respectively. Then, the method of computing the determinant of given complex quaternion and complex split quaternion matrices has been proposed. Although, the determinant properties, conjugate products, special matrices of complex quaternion and complex split quaternion matrices were investigated with the similar methods, but different results were obtained due to the difference of the basis elements of these quaternion matrices.

\section{REFERENCES}

[1] Y. Alagöz, K. H. Oral, and S. Yüce, "Split quaternion matrices.” Miskolc Math. Notes, vol. 13, no. 2, pp. 223-232, 2012, doi: 10.18514/MMN.2012.364.

[2] H.-D. Ebbinghaus, H. Hermes, F. Hirzebruch, M. Koecher, K. Mainzer, J. Neukirch, A. Prestel, and R. Remmert, Numbers. With an introduction by Klaus Lamotke. Translated by H. L. S. Orde. Edited by John H. Ewing. Paperback ed., paperback ed. ed. New York etc.: Springer-Verlag, 1991, vol. 123, doi: 10.1007/978-1-4612-1005-4.

[3] M. Erdoğdu and M. Özdemir, "On complex split quaternion matrices." Adv. Appl. Clifford Algebr., vol. 23, no. 3, pp. 625-638, 2013, doi: 10.1007/s00006-013-0399-z.

[4] K. Gürlebeck, K. Habetha, and W. Sprößig, Application of holomorphic functions in two and higher dimensions. Basel: Birkhäuser/Springer, 2016. doi: 10.1007/978-3-0348-0964-1.

[5] M. L. Mehta, Random matrices. Boston, MA: Academic Press, Inc., 1991.

[6] B. Rosenfeld, Geometry of Lie groups. Dordrecht: Kluwer Academic Publishers, 1997.

[7] J. P. Ward, Quaternions and Cayley numbers: algebra and applications. Dordrecht: Kluwer Academic Publishers, 1997. doi: 10.1007/978-94-011-5768-1.

Authors' addresses

Y. Alagöz

Yildiz Technical University, Department of Mathematics, 34220 Istanbul, Turkey

E-mail address: ygulluk@yildiz.edu.tr 
G. Özyurt

Yildiz Technical University, Department of Mathematics, 34220 Istanbul, Turkey

E-mail address: gozdeozyurt1@gmail.com 